\title{
A Sample of GeMS Calibrations and Control Schemes
}

\author{
Francois Rigaut $^{1, \mathrm{a}}$, Benoit Neichel ${ }^{1}$, Matthieu Bec ${ }^{1}$, Maxime Boccas ${ }^{1}$, Aurea Garcia-Rissmann ${ }^{1}$, and \\ Damien Gratadour ${ }^{2}$ \\ 1 Gemini Observatory, c/o AURA, Casilla 603 La Serena, Chile \\ 2 Observatoire de Paris-Meudon, 2 Place Jules Janssen 92195 Meudon-CEDEX France
}

\begin{abstract}
The Gemini MCAO system, GeMS, is currently undergoing integration and test at the Gemini South base facility. GeMS has been in the work since 2001, and is finally reaching a state in which all the subsystems, except for the 50W laser, have been received, integrated and, in the large part, tested. In this paper, after a brief description of the system, we describe selected methods and results that we have obtained during the on-going integration period.
\end{abstract}

\section{Introduction: GeMS in numbers}

GeMS is the Gemini Multi-conjugate adaptive optics System. It has been described elsewhere [1]. GeMS is currently undergoing integration and test at the Gemini South base facility, with a first light planned for mid-2010. The main components of GeMS are:

- 5 laser guide star (LGS) Shack-Hartmann wavefront sensors (WFS), 16x16 subapertures.

- 3 Deformable Mirrors (DM) totaling 917 actuators (684 active, 233 extrapolated), conjugated optically to $0,4.5$ and $9 \mathrm{~km}$ above the telescope.

- 3 avalanche photodiodes (APD) based quadcell natural guide star (NGS) Tip-Tilt (TT) WFS

- 1 NGS slow focus WFS (SFS)

- 1 x 50W laser divided in 5 x 10W beams forming a LGS constellation. The LGS are placed on the sky at the corner and center of a 60 arcsec square

- Two dedicated instruments:

- GSAOI (4k2 NIR imager), 80” FoV (20mas pixels) [2]

- Flamingo II (NIR MOS), 2' FoV [3]

- Many subsystems: All-Sky-Camera, Safety systems, infrastructure, laser(s), Beam Transfer Optics (BTO), Laser Launch Telescope (LLT), etc, that are not described here. Suffice to say that a MCAO system is more than just the AO optical bench. All these "side" subsystems represent more than half the cost and the workload.

Figure 1 shows the overall GeMS diagram. It has been included for reference and to illustrate the complexity of this system. A more complete description of the system can be found in $[4,5]$.

\section{Synthetic matrices and how to use them}

The almost universal approach in field AO systems is to use measured interaction matrices (iMat).

An alternative approach [6] is to completely simulate the system and use synthetic iMats. In the following, we briefly compare both approaches.

Drawbacks and advantages of using measured iMats include:

$\uparrow$ They include all the actual system characteristics and idiosyncrasies, such as WFS response and gain, DM actuator gains, optical misalignment, etc...

$\downarrow$ They contain noise, even if special techniques like Hadamard, can substantially reduce it.

\footnotetext{
a e-mail: frigaut@gemini.edu
} 


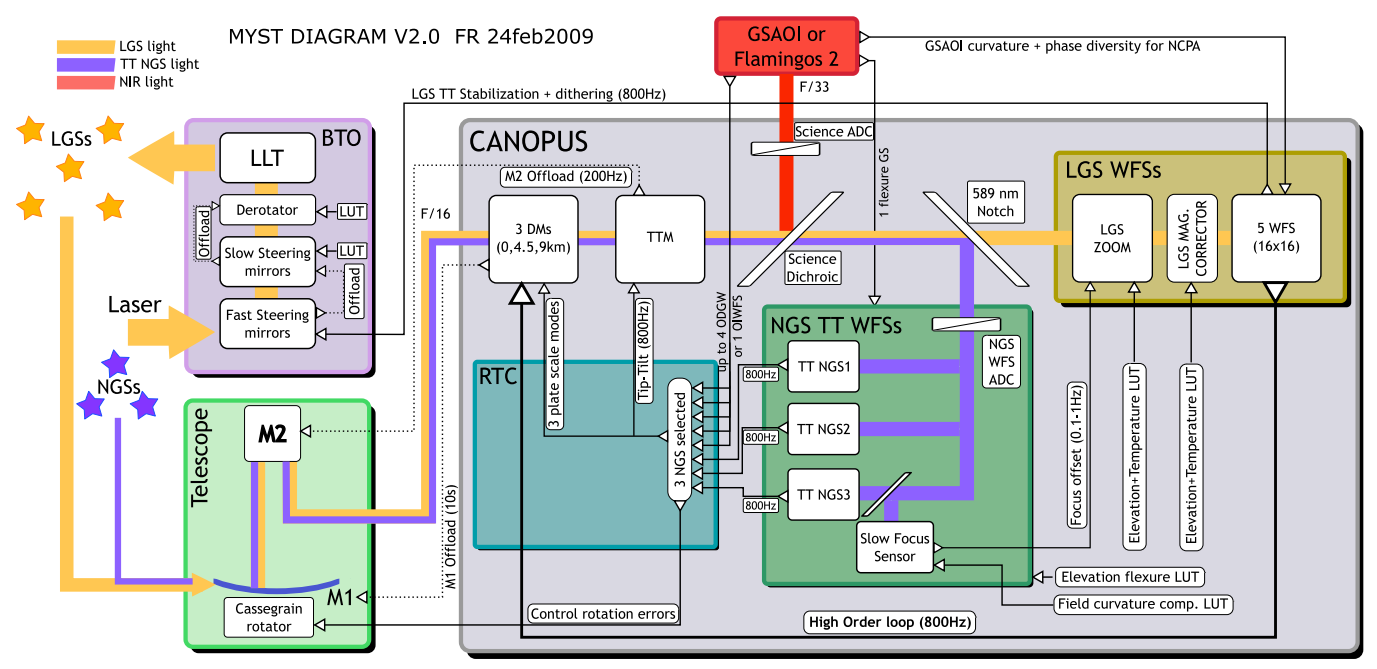

Fig. 1. GeMS synaptic diagram, that shows components, loops and offloads

$\downarrow$ It can be argued that to fully include real system effects, the iMat would need to be recorded on the sky, that is, in the actual conditions it will be used, and a lot of work has been done in that direction [6]. Whether this is done or not, it consumes valuable time.

Drawbacks and advantages of synthetic iMats include:

$\uparrow$ They are noiseless. For systems with a large number of degrees of freedom (the iMat is $684 \times 2040$ in GeMS), the stability and accuracy of the inversion process critically depends on the signal tonoise of the input iMat.

$\uparrow$ They can be readily pre-computed. This may be very relevant if the iMat is dependent on operational parameters ${ }^{1}$.

$\uparrow$ They can be hooked to numerical simulations. This turned out to be convenient with GeMS to check the sanity and level of performance of the actual system. In GeMS, we use the simulation tool for many purposes: generating iMats, but also hooking to our diagnostics WFS (see Section 4), implementing all kinds of diagnostics, projecting on modes, generating disturbances, etc... The numerical simulation code is also hooked to the high level control software and can be used as a MCAO simulator.

$\uparrow$ It is possible to include DM and WFS gains, and misregistration.

$\downarrow$ They do not include other system idiosyncrasies, or in general can not reproduce any system aspects and behavior that is not understood.

Note that neither experimental nor synthetic matrices can compensate for non-linearities, by essence of the linear matrix relationship between measurements and DMs which is used in the vast majority of systems. Non-linearities can be accounted for and compensated, but has no direct relationship with this experimental versus synthetic discussion.

Synthetic matrices can not be generated without precautions. A necessary set of conditions is:

- DM actuator gains and, if necessary, WFS subaperture gains (centroid gains, if using quadcells) have to be calibrated and factored into the synthetic iMat (or the actuator/measurement vector gain-compensated in the real-time software). In GeMS, both the WFS measurement and the DM command vector are compensated for gain (see Section 5 for an overview of the LGS centroid gain issue).

1 An LGS MCAO/LTAO/MOAO iMat, for instance, will generally depend on the telescope elevation: the LGS/sodium layer range varies with elevation and thus the beam patch on the DMs will change 

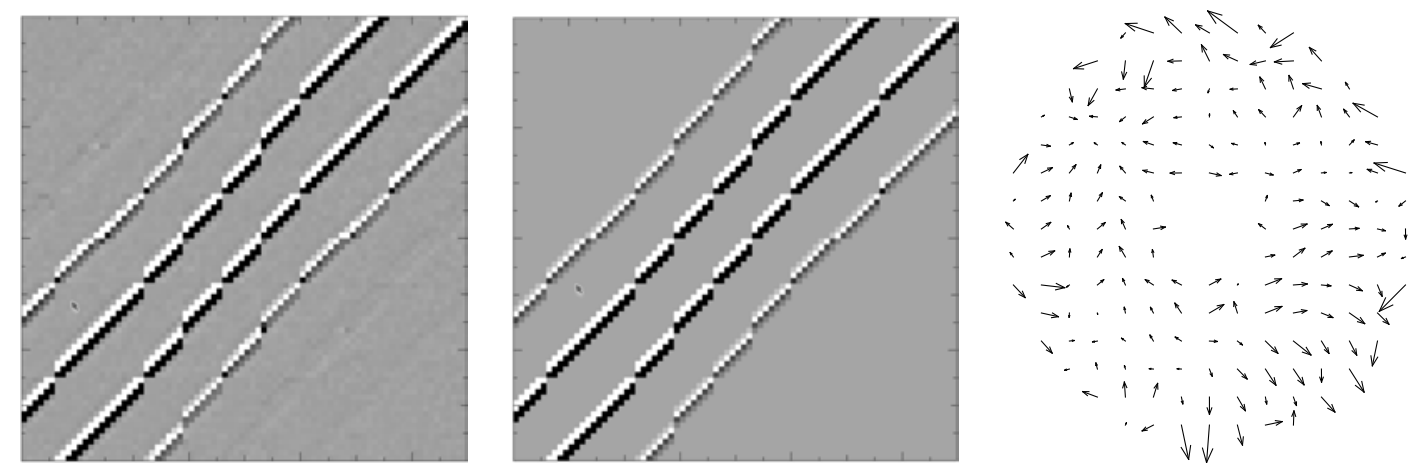

Fig. 2. Measured (left) versus synthetic (center) interaction matrix. Right: DM0 actuators to WFS0 registration map. Largest vector is $4.5 \%$ of a subaperture.

- The DMs influence functions have to be adjusted to match the actual ones. For GeMS, we chose a relatively simple functional form (gaussian $\times$ sinc) and we minimized the distance between an experimental iMat and the synthetic iMat, leaving the influence function parameters and the misregistration on a per actuator basis as free parameters.

- Misregistration has to be factored in. In GeMS, we have a calibration sequence to check and adjust the DMs misregistration, on a per WFS basis. It turns out that the pupil distortion is small enough that this step is sufficient (no correction for local distortion).

- Generally speaking, it is best to include as many effects as possible, which generally needs an advanced numerical simulation tool. In GeMS case, we use yao, an adaptive optics package written in yorick (http://www.maumae.net/yao/). Yao features of interest in the frame of generating the iMats include among others a diffractive Shack-Hartmann (and curvature) WFS model, adjustable influence function parameters, misregistration.

In the end, the criteria to judge the acceptability of the synthetic matrix approach will be a mix of performance on bright and faint stars, weighted (assuming there's a loss) against practical considerations like reduced day or night-time calibration overheads. For now, synthetic iMats are being used every day in the laboratory and have proven stable. They provide good performance levels, under high and low illumination conditions.

Figure 2 (left and center) shows a sample comparison between a measured versus a synthetic iMat for GeMS.

In summary, both experimental and synthetic approaches have their drawbacks and advantages. It is likely that the use of synthetic matrices will increase in the future, as (1) systems become larger (in terms of number of actuators) and (2) components, alignment and assembly becomes more refined, leading to system behaving closer to what is expected and what can be modeled.

\section{DMs/WFSs registration}

To avoid loop instabilities and maximize performance, it is well known that it is important to maintain a good registration between the DM actuators and the WFS subapertures. In a Fried geometry, loop instabilities will start to appear for misregistration of 15 to $20 \%$ of an actuator pitch. This becomes more critical, in terms of overall pupil registration, for systems with a large number of actuators. In a real system, there are essentially three causes of misregistration:

- Flexures,

- Encoder malfunction. Many AO system include some kind of field selection, or in GeMS case, zoom mechanism to compensate for the LGS range variations, between the DM(s) and WFS(s). These mechanisms can loose steps (regardless of what engineers will tell you),

- Local pupil distortion. 
In GeMS, we have two calibration sequences to check for misregistration: a quick one that will check for a global misregistration (X,Y, magnification and rotation) by pushing 8 actuators around the pupil and a more thorough one that measures the misregistration of all actuators by comparing the measured iMat with the synthetic one (see Section 2). Figure 2 (right) gives the result of the latter, i.e. a map of actuators to WFS subapertures registration. There is no systematic effect. The rms of this vector field is $1.2 \%$ in both $\mathrm{X}$ and $\mathrm{Y}$, with an extremum value of $4.5 \%$. Over all WFSs, the rms is typically below $2 \%$ and the extrema typically $5 \%$ (excluding the edge actuators, for which the estimate is necessarily more noisy due to the lack of information outside of the pupil area).

The effect of flexures has also been evaluated, and found to induce a misregistration of up to $25 \%$ of a subaperture at a roll angle of 55 degrees (close to the maximum of 60 degrees). This, however, will be compensated using a Look-UP Table.

\section{Compensation of the Non-Common Path Aberrations}

Dealing with Non-Common Path Aberrations (NCPA) in an MCAO system is more complex than in a classical, single conjugated AO system. The fundamental difference is that, in an MCAO system, the static aberrations have to be compensated simultaneously over the entire output focal plane. The basic principle remain the same though: the problem consists in finding the set of slopes (and thus the set of actuator offsets) that will minimize the static phase error in the output/science focal plane. As far as the science path is concerned, the fact that only the DMs can be used to compensate for aberrations has the consequence that static aberrations induced by optics that are alitude-conjugated outside of the range spanned by the DMs are not correctable, because it can not be compensated for the whole field simultaneously ${ }^{2}$. This is an important consideration for the optical design and components specification of a MCAO system.

\subsection{Setup and NCPA in one direction only}

The NCPA setup has been described in details in [5]. Briefly: a Diagnostic 24x24 Shack-Hartmann WFS (DWFS) has been installed at the science focal plane for laboratory work. This DFWS can be moved and sequentially acquire phase measurements at arbitrary positions in the focal plane. The DWFS has been calibrated using solely its plate scale. A house developed GUI+yorick software (WAY) is used to reconstruct the phase and to provide the Zernike coefficients. To within a few percent, this calibration is consistent with the modes produced by GeMS, in response to slope offsets.

Before taking care of the full field of view NCPA compensation, we chose to start by tackling the NCPA in a single, arbitrary direction. We followed the usual, iterative method:

1. Measure the raw aberrations with the DWFS

2. Use our adaptive optics simulation model (see Section 2) to compute the LGS WFS slopes corresponding to the measured Zernike coefficients

3. Apply the slope offsets, close the loop

4. Restart at (1) and update offsets with residuals

It turned out that the model accuracy is such that one iteration is generally sufficient. Raw $\mathrm{H}$ band Strehl ratios, depending on the field position, range from $5 \%$ to $75 \%$ (dominated by astigmatism). A single iteration of NCPA results in $\mathrm{H}$ band Strehl ratios at or above $99 \%$. This proves that we understand the calibration and linearity issues of the DWFS and of the LGSWFSs (including centroid gains) and it validates, at least in part, our numerical simulation tools.

Note that this setup is only be used in the laboratory. On the telescope, we will use GSAOI images. GSAOI has been built to provide extra-focal images -using 2 lenses in its utility wheel- from which phase estimates are derived simultaneously at many points in the field of view (from several stars). They are fed to GeMS in the same fashion as the current DWFS measurements.

\footnotetext{
2 quadratic modes are an exception to that rule
} 


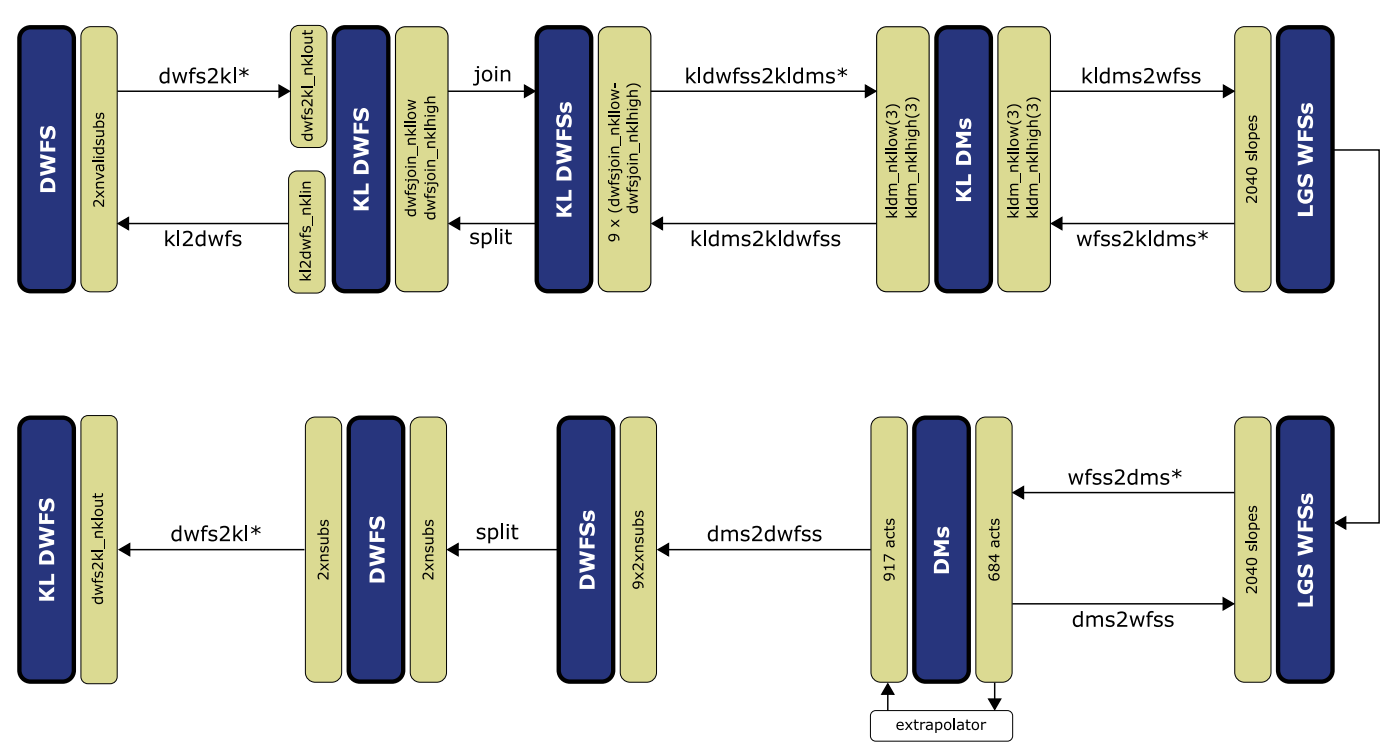

Fig. 3. NCPA control block diagram.

\subsection{Tomographic control and results}

The extension of the above described process to $\mathrm{N}$ points requires to solve a tomographic problem. This is relatively well known, at least on paper: the basic steps are (1) solve the tomography on $\mathrm{N}$ layer, (2) project on a layer subset (available DMs) and (3) project on the WFS slopes. Like in many problems, the devil is in the details, so we will further describe our implementation.

Figure 3 present a block diagram of the various components (blue boxes) and projections/operations (arrows). The upper row shows the operations that lead, from the DWFS slopes, to the LGSWFS offset slopes. The bottom row shows the way LGSWFS offsets are projected back to the DWFS to predict the expected performance. Notations are the same as in this paper (DM/WFS), with the addition of KL, which stands for Karhuenen-Loeve. The operators are all matrix multiplies, and their names are fairly self-explanatory, e.g. kldwfss2kldms means KL DWFSs ( 9 of them) to KL DMs ( 3 of them). In random order, here are some comments about our findings:

- Although we could have used the DWFS slope basis as an input, then deduce DM actuator commands, then projected in LGS WFS slopes, we found this process unstable (both with TSVD and MMSE). One of the issues is the sheer size of the matrix to invert to solve the tomography: we have 9 DWFS pointings, each one with 912 slopes, leading to a $8208 \times 917$ matrix, which proved difficult to invert using standard techniques (numerical issues).

- Because NCPA are likely to be dominated by low order modes, we decided to restrict the DWFS measurements to a finite number of modes. We found that the KLs showed better behavior than the Zernikes -the former have significantly smaller edge slopes than the latter-, leading to more accurate reconstruction over 100 modes or more. DWFS slopes where thus projected on KL, restricting the order of the tomographic matrix. The tomography was solved using KL DMs, and then projected to LGS WFS slopes. All of the projection and tomography matrices used in this process were computed using our adaptive optics simulation tool yao.

Results have been encouraging: In three iterations, we reach a $\mathrm{H}$ band static Strehl ratio of $96 \% \pm$ $1 \%$ (all Strehl ratio were measured by the DWFS using 120 Zernike modes). Two iterations are needed to compensate the high order aberrations, and a third one is used controlling only astigmatism, which make most of the initial aberrations. Additional iterations do not provide further improvements, which tends to indicate the presence of $50 \mathrm{~nm}$ rms aberrations that can not be corrected, probably because it is located outside of the altitude range that can be compensated by the system $(0-9 \mathrm{~km})$. This performance exceeds the science requirements $(94 \% \pm 1 \%)$. 


\section{LGS WFS centroid gains}

Each GeMS LGS WFS uses a EEV CCD (80x80), with 16x16 subapertures. Each subaperture on the CCD use $2 \times 2$ pixels (quadcell), with one row/column of guard pixels on each side (thus uses $16 \times 4$ $=64$ pixels). Special waveforms are loaded that only read the valid pixels (816) at a frame rate of $800 \mathrm{~Hz}$, thus $4 \mu \mathrm{s} /$ pixels (there are four amplifiers). In the laboratory, we achieve regularly $3.5 \mathrm{e}-\mathrm{read}$ out noise, often down to 3.3e-, with all the electronics turned on.

The use of quadcells implies to calibrate the centroid gain for each subaperture ${ }^{3}$. We use two method to estimate the centroid gains:

- In the laboratory, we simply use the TT mirror to sweep the spots in front of the quadcell, fit a simple functional form, and get the slope at origin.

- While the loop is closed, it is obviously impossible to use the method above. We have implemented and tested a dithering method, with which we are accustomed as it has been used in the Gemini North adaptive optics system Altair [7]. The LGS are dithered in a fast circular motion. We use a 100mas radius (this value might be reduced to 50 mas in the future, but even 100 mas will be small compared to the expected 1 arcsec or larger LGS spot FWHM), at a frequency still to be fine tuned, between 30 and $200 \mathrm{~Hz}$. When on sky, the dither will be induced by TT platforms in the laser launch path. In the laboratory, we use the TT mirror.

Both methods compare very well. They give similar centroid gain estimates to within a few percent.

\section{Conclusion}

GeMS is the first instrument of its kind: a Multi-Conjugate adaptive optics system using Laser Guide Stars. Integration in the laboratory has revealed many unexpected, and confirmed expected issues. This has called for innovative solutions, of which we have presented a subset. We have presented arguments in favor of synthetic interaction and control matrices, which have been used with success in the laboratory. We reported on the resolution and performance of the compensation of static NCPAs. $\mathrm{H}$ band Strehl ratio of $96 \%$ have been obtained over the entire science output focal plane. We also commented on other critical aspects of GeMS calibrations: the DM/WFS registration and the LGS WFS centroid gain controls. The integration follows its course in the laboratory at the Gemini South instrument integration hall. Recently, we have interfaced a CCD camera in parallel to the DWFS presented above. This will allow us to start investigating the dynamical performance of the bench and rank our various reconstructors [4],- validate our synthetic iMat approach, and extend/check the NCPA analysis. The latest schedule puts the first light in June 2010.

The Gemini Observatory is operated by the Association of Universities for Research in Astronomy, Inc., under a cooperative agreement with the NSF on behalf of the Gemini partnership: the National Science Foundation (United States), the Science and Technology Facilities Council (United Kingdom), the National Research Council (Canada), CONICYT (Chile), the Australian Research Council (Australia), Ministerio da Ciencia e Tecnologia (Brazil), and Ministerio de Ciencia, Tecnologia e Innovacion Productiva (Argentina).

\section{References}

1. M. Bec et al., Proc. SPIE, Vol. 7015 (2008)

2. Peter McGregor et al., Proc. SPIE, Vol. 5492 (2004)

3. S. Eikenberry et al., Proc. SPIE, Vol. 7014 (2008)

4. B. Neichel, F. Rigaut, M. Bec \& Aurea Garcia Rissmann, this conference

5. A. Garcia Rissmann, F. Rigaut, M. Bec, et al., this conference

6. S. Oberti, F. Quirós-Pacheco; S. Esposito, Proc. SPIE Volume 6272 (2006)

7. D. Gratadour \& F. Rigaut, in Adaptive Optics: Analysis and Methods, OSA, paper PMA4 (2007)

3 A quadcell signal is unitless; the centroid gain will convert it in meaningful units, e.g. arcsec 